Unfallchirurg 2013 $\cdot 116: 294-295$

DOI 10.1007/s00113-012-2344-3

Online publiziert: 24. März 2013

(c) Springer-Verlag Berlin Heidelberg 2013

P. Biberthaler ${ }^{1} \cdot$ H. Lill ${ }^{2}$

${ }^{1}$ Klinik und Poliklinik für Unfallchirurgie, Klinikum rechts der Isar, Technische Universität München

${ }^{2}$ Klinik für Unfall- und Wiederherstellungschirurgie, Diakoniekrankenhaus Friederikenstift gGmbH, Hannover

\title{
Arthroskopisch unterstütztes Frakturmanagement
}

Bei der Patellafraktur oder osteochondralen Frakturen bekommt der Arthroskopie des Kniegelenks wieder eine zunehmende Bedeutung.

Die Arthroskopie bei akuten distalen, intraartikulären Radiusfrakturen hat sich im klinischen Alltag nicht etabliert, wird jedoch bei bestimmten Fragestellungen und begleitenden Kapsel-Band-Verletzungen eingesetzt. Hierbei eröffnen sich dann interessante therapeutische Aspekte.

\section{》) Aktueller Stand der therapeutischen Möglichkeiten für die Versorgung von Gelenkfrakturen mittels arthroskopisch unterstützter Techniken}

Der Arthroskopie bei proximalen Humerusfrakturen kommt eine zunehmende Bedeutung zu, da auch Möglichkeiten der „Weichteilarthroskopie“ gegeben sind. Dies betrifft in diesem Zusammenhang in erster Linie die Reposition und Fixation von Tuberculum-majus-Frakturen. Die arthroskopische Visualisierung der Fraktur verbessert in Kombination mit der BV-Durchleuchtung die Darstellung und die exakte Reposition der Fraktur. Moderne Fixationstechniken (z. B. das „suturebridging") konnten erst arthroskopisch entwickelt werden. Die Begleitverletzungen, wie z. B. die des Bizepssehnenkomplexes, können in gleicher Sitzung arthroskopisch therapiert werden. Die arthroskopisch gestützte Insertion von geraden
Nägeln bei subkapitalen 2-Segment-Frakturen des Humerus ist eingeführt worden und weist in Bezug auf die Kompromittierung der Rotatorenmanschette alle Vorteile der Minimalinvasivität auf.

Diesen Vorteilen steht der teilweise erhebliche technische Aufwand, die teilweise deutlich verlängerte Operationszeit und die flachere Lernkurve des Operateurs gegenüber, welche durch zunehmende Erfahrung des Operateurs und Teams ausgeglichen werden können.

Auch arthroskopisch gestützte Frakturrepositionen des Pilon tibiale mit Arthroskopie des ventralen Kompartiments des oberen Sprunggelenks (OSG) sind möglich und bieten bei bestimmten Frakturen entsprechende Vorteile - in diesen Fällen insbesondere die Gelenklavage, die Entfernung kleinerer Fragmente aus dem Frakturspalt und die visualisierte Reposition. Dies gilt gleichermaßen für große Volkmann-Dreiecke bei OSGLuxationsfrakturen über die Arthroskopie des hinteren Kompartiments. Die „offene“ Arthroskopie des unteren Sprunggelenks (USG) bei Gelenkfrakturen des Kalkaneus hat sich mittlerweile etabliert und bietet ein gutes Hilfsmittel, neben der 3D-BVDarstellung, zur intraoperativen Repositionskontrolle.

Somit ist das Thema der arthroskopischen Frakturversorgung aktueller denn je. In dem Leitthemenheft haben Mitglieder des Komitees TRAUMA der AGA (Karl-Heinz Frosch, Christian Schoepp, Christine Voigt) als ausgewiesene Spezialisten auf diesem Gebiet Indikationen, Durchführungen sowie Gren- 
zen und Gefahren wissenschaftlich, aber dennoch praxisnah dargestellt.

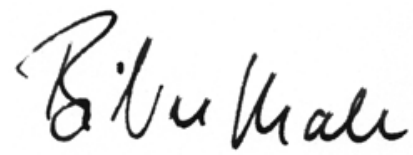

P. Biberthaler

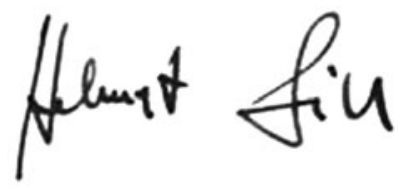

H. Lill

(Vorsitzender des Komitees TRAUMA der AGA)

\section{Korrespondenzadressen}

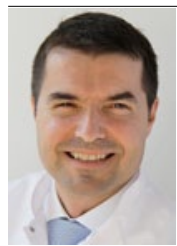

Prof. Dr. P. Biberthaler

Klinik und Poliklinik für

Unfallchirurgie,

Klinikum rechts der Isar,

Technische Universität

München

Ismaninger Straße 22,

81675 München

peter.biberthaler@mri.tum.de

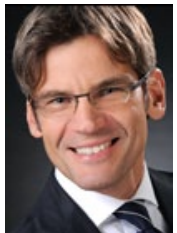

Prof. Dr. H. Lill

Klinik für Unfall- und

Wiederherstellungschirurgie,

Diakoniekrankenhaus

Friederikenstift gGmbH

Humboldtstraße 5,

30169 Hannover

helmut.lill@ddh-gruppe.de
Der Orthopäde bietet Ihnen jeden Monat umfassende und aktuelle Beiträge zu interessanten Themenschwerpunkten aus allen Bereichen der Orthoäpdie. In mehreren Übersichtsartikeln wird ein Sachgebiet vertiefend dargestellt. Möchten Sie ein bereits erschienenes Heft nachbestellen? Einzelne Ausgaben können Sie direkt bei unserem Kundenservice zum Preis von je EUR 35,- zzgl. Versandkosten beziehen:

\section{Heft 3/2013}

- Evaluation des sagittalen Profils nach operativer Therapie der thorakalen adoleszenten idiopathischen Skoliose Lenke Typ 1

- Elektromagnetische Navigation transpedikulärer Punktionen

- Prothetik bei proximalen Humerusfrakturen

- Biokompatibilität von Polymer-Glaskeramik-Zement Cortoss ${ }^{\circledast}$

- Therapierelevante Bildgebung in der modernen Arthrosebehandlung am Beispiel des Schultergelenks

- CME: Das Knochenmarködemsyndrom (KMÖS)

\section{Heft 4/2013}

- Grundlagen zur tribologischen Analyse von Endoprothesen

- Einsatzgebiete der numerischen Simulation in der muskuloskelettalen Forschung

- Möglichkeiten der biomechanischen Charakterisierung von Knorpelgewebe

- Defektmodelle für die Gelenkknorpelregeneration im Großtiers

- CME: Bildwandler-gestützte Injektionstechniken an der Lendenwirbelsäule

So erreichen Sie unseren Kundenservice:

Springer Customer Service Center GmbH

Kundenservice Zeitschriften

Haberstr. 7

69126 Heidelberg

Tel.: +49 6221 345-4303

Fax: +49 $6221345-4229$

E-Mail: leserservice@springer.com

\section{www.DerOrthopaede.de}

